\title{
Glycosyltransferase - a specific marker for the discrimination of Bacillus anthracis from the Bacillus cereus group
}

Wonyong Kim, ${ }^{1,2}$ Ji-Yeon Kim, ${ }^{1}$ Sung-Lim Cho, ${ }^{1,2}$ Sun-Woo Nam,
Jong-Wook Shin, ${ }^{4}$ Yang-Soo Kim ${ }^{5}$ and Hyoung-Shik Shin ${ }^{6}$

Correspondence

Wonyong Kim

kimwy@cau.ac.kr

Received 20 September 2007

Accepted 12 November 2007

\author{
${ }^{1}$ Department of Microbiology, Chung-Ang University College of Medicine, Seoul, Republic of Korea \\ ${ }^{2}$ Research Institute for Translational System Biomics, Chung-Ang University College of Medicine, \\ Seoul, Republic of Korea \\ ${ }^{3}$ Korea Health Industry Development Institute, Seoul, Republic of Korea \\ ${ }^{4}$ Department of Internal Medicine, Chung-Ang University College of Medicine, Seoul, Republic of \\ Korea \\ ${ }^{5}$ Department of Radiology, Chung-Ang University College of Medicine, Seoul, Republic of Korea \\ ${ }^{6}$ Department of Periodontology, Wonkwang University College of Dentistry, Iksan, Republic of \\ Korea
}

Bacillus anthracis, the aetiological agent of anthrax, has been taxonomically classified with the Bacillus cereus group, which comprises B. cereus, Bacillus thuringiensis, Bacillus mycoides, Bacillus pseudomycoides and Bacillus weihenstephanensis. Although the pathogenesis and ecological manifestations may be different, $B$. anthracis shares a high degree of DNA sequence similarity with its group member species. As a result, the discrimination of $B$. anthracis from its close relatives in the $B$. cereus group is still quite difficult. Suppression subtractive hybridization (SSH) was performed to search for genomic differences between a $B$. anthracis Korean isolate CR and the most closely related $B$. cereus type strain KCTC $3624^{\top}$. Two-hundred and five $B$. anthracis CR clones obtained by SSH underwent Southern hybridization, and comparative sequences were analysed using the BLAST program from the National Center for Biotechnology Information (NCBI). Subsequently, primer sets based on the glycosyltransferase group 1 family protein gene specific to $B$. anthracis were designed from the sequences of subtracted clones, and their specificities were evaluated using eight $B$. anthracis, 33 B. cereus, 10 B. thuringiensis, six B. mycoides, one B. pseudomycoides, one B. weihenstephanensis and 19 strains from 11 other representative Bacillus species. PCR primers specific for the glycosyltransferase group 1 family protein gene did not amplify the desired products from any of the Bacillus strains under examination, except $B$. anthracis alone. These findings may be useful in the future development of efficient diagnostic tools for the rapid identification of $B$. anthracis from other members of the $B$. cereus group.

\section{INTRODUCTION}

The Bacillus cereus group comprises Bacillus anthracis, Bacillus cereus, Bacillus thuringiensis, Bacillus mycoides, Bacillus pseudomycoides and Bacillus weihenstephanensis (Ash et al., 1991; Lechner et al., 1998; Nakamura, 1998). B. anthracis, B. cereus and B. thuringiensis were the first three species described (Priest et al., 2004), and they are not easily distinguishable from one other on the basis of phenotypic or genotypic traits. Methods used to discriminate B. cereus

Abbreviations: AFLP, amplified fragment length polymorphism; SSH, suppression subtractive hybridization. group species based on phenotypic traits include motility, biochemical testing (Logan et al., 1985; Odendaal et al., 1991), antibiotic susceptibility testing (Mohammed et al., 2002), direct fluorescent antibody staining of capsule and cell wall polysaccharide (Sastry et al., 2003), lysis of colonies by gamma phage (Logan et al., 1985; Schuch et al., 2002), and animal virulence testing (Odendaal et al., 1991). Although these species share a high degree of genetic relatedness, attempts have been made to distinguish $B$. cereus group member strains as demonstrated by wholegenome DNA-DNA hybridization (Read et al., 2003), 16S and 23S rRNA gene sequencing (Ash et al., 1991; Ash \& 
Collins, 1992), gyrB-gyrA (Harrell et al., 1995) or 16S-23S rDNA intergenic spacer region comparisons (Daffonchio et al., 1998; Cherif et al., 2003), PFGE analysis (Carlson et al., 1994), variable number tandem repeat mapping (VNTR; Andersen et al., 1996; Keim \& Smith, 2002), BOXPCR fingerprinting (Kim et al., 2002), and multilocus sequence typing (MLST) (Helgason et al., 2000, 2004; Priest et al., 2004).

This diverse range of methods has failed to reveal consistent differences between isolates to warrant segregating $B$. anthracis, $B$. cereus and $B$. thuringiensis into discrete species (Read et al., 2003); separate species status is maintained because of their pathogenic traits (Priest et al., 2004). Although PCR-based methods can differentiate vaccine or fully virulent $B$. anthracis genotypes, there is difficulty in distinguishing plasmid-cured $B$. anthracis or near-neighbour species. Furthermore, these techniques lack assay specificity for the detection of the $B$. anthracis chromosome (Daffonchio et al., 1999) and can yield falsepositive results (Ellerbrok et al., 2002).

Suppression subtractive hybridization ( $\mathrm{SSH}$ ) has been used to analyse genomic differences (Radnedge et al., 2003) and compare pathogenic and non-pathogenic bacterial strains to identify possible virulence factors among bacterial strains (Agron et al., 2002). The advantage that SSH has over microarray DNA-DNA hybridization (Volokhov et al., 2004) is that the latter identifies only those genes present in the reference genome and not genes which are absent (Jones et al., 2006). The objective of this study was to use
SSH to search for genomic differences between the $B$. anthracis Korean isolate CR and the most closely related $B$. cereus type strain KCTC $3624^{\mathrm{T}}$, and to identify sequences useful for rapid B. anthracis identification and diagnosis.

\section{METHODS}

Bacterial strains. A total of 78 bacterial strains were used in this study and are listed in Table 1. B. anthracis ATCC $14578^{\mathrm{T}}$, ATCC 14185 and ATCC 14186 were obtained from the American Type Culture Collection (ATCC), and Korean isolates of B. anthracis CR and $\mathrm{BC}$ were provided by the National Veterinary Research and Quarantine Service (Anyang, Korea). The recent outbreak strain of $B$. anthracis $\mathrm{CR}$ was isolated from beef eaten by a patient who died from gastrointestinal anthrax in 2000, and B. anthracis CAU 1, CAU 2 and CAU 3 were isolated in our laboratory from patients in 1994. The remaining 70 Bacillus strains comprised isolates from our laboratory and strains obtained from the Korean Collection of Type Cultures (KCTC; Taejeon, Korea), the Korean Culture Center of Microorganisms (KCCM; Seoul, Korea) or the ATCC.

Genomic DNA preparation. Each bacterial strain was grown on brain heart infusion agar (Difco) at $28{ }^{\circ} \mathrm{C}$ for $16 \mathrm{~h}$, and bacterial genomic DNA was prepared by employing the cetyltrimethylammonium bromide method (Ausubel et al., 1993). Purified DNA was dissolved in sterile water with $40 \mu \mathrm{g} \mathrm{ml}^{-1}$ RNase, and quantified using an MBA 2000 spectrophotometer (Perkin-Elmer) at a wavelength of $260 \mathrm{~nm}$.

Construction of subtracted genomic DNA libraries by SSH. Genes unique to B. anthracis were identified by SSH using B. anthracis CR as tester DNA and B. cereus KCTC $3624^{\mathrm{T}}$ as driver DNA. SSH was

Table 1. Bacillus species $(n=78)$ used in this study

\begin{tabular}{|c|c|c|}
\hline Group & Species & Strain \\
\hline \multirow{6}{*}{$\begin{array}{c}\text { B. cereus } \\
(n=59)\end{array}$} & B. anthracis & ATCC $14578^{\mathrm{T}}$, ATCC 14185, ATCC 14186, Korean isolates CR, BC, CAU 1, CAU 2, CAU 3 \\
\hline & B. cereus & $\begin{array}{l}\text { KCTC } 3624^{\mathrm{T}} \text {, KCTC 1012, KCTC 1013, KCTC 1014, KCTC 1094, KCTC 1661, KCTC 3062, ATCC } \\
\text { 10702, ATCC 13061, ATCC 21769, ATCC 21771, ATCC 25621, ATCC 27348, Korean isolates } \\
\text { WY1, WY2, WY3, WY4, WY5, WY6, WY7, WY8, WY9, WY10, WY11, WY12, WY13, WY14, } \\
\text { WY15, WY16, WY17, WY18, WY19, WY20 }\end{array}$ \\
\hline & B. thuringiensis & KCTC $1034^{\mathrm{T}}$, KCTC 1507, KCTC 1509, KCTC 1519, KCTC 3452, Korean isolates C1, C2, C3, C4, C5 \\
\hline & B. mycoides & KCTC $3453^{\mathrm{T}}$, ATCC 10206, ATCC 19647, ATCC 21929, ATCC 23258, KCCM 40260 \\
\hline & B. pseudomycoides & KCTC $3862^{\mathrm{T}}$ \\
\hline & B. weihenstephanensis & KCTC $3975^{\mathrm{T}}$ \\
\hline \multirow{12}{*}{$\begin{array}{l}\text { Other } \\
\text { Bacillus } \\
(n=19)\end{array}$} & Bacillus circulans & KCTC $3347^{\mathrm{T}}$ \\
\hline & & \\
\hline & Bacillus coagulans & KCTC $3625^{\mathrm{T}}$, KCTC 1015 \\
\hline & Bacillus licheniformis & KCTC $1918^{\mathrm{T}}$ \\
\hline & Bacillus pumilus & KCTC $3348^{\mathrm{T}}$ \\
\hline & Bacillus sphericus & KCTC $3346^{\mathrm{T}}$ \\
\hline & Bacillus subtilis & КСТС $3135^{\mathrm{T}}$, КСТС 1021, КСТС 1022, КСТС 1028, КСТС 1691, КСТС 3014 \\
\hline & Bacillus atrophaeus & KCTC $3701^{\mathrm{T}}$ \\
\hline & Bacillus mojavensis & KCTC $3706^{\mathrm{T}}$ \\
\hline & Bacillus vallismortis & KCTC $3707^{\mathrm{T}}$ \\
\hline & Bacillus amyloliquefaciens & KCTC $1660^{\mathrm{T}}$, КCTC 3002, КССM 40764 \\
\hline & Bacillus stearothermophilus & KCTC $1752^{\mathrm{T}}$ \\
\hline
\end{tabular}


performed using the PCR-Select Bacterial Genome Subtraction kit (Clontech) according to the manufacturer's instructions, with some modifications. Tester and driver genomic DNA $(1 \mu \mathrm{g})$ were digested separately with the restriction enzyme RsaI (Roche Diagnostics), generating fragments between 500 and $2000 \mathrm{bp}$ in size (data not shown). Then, digested tester DNA was subtracted and amplified by PCR. Tester-specific fragments were subsequently cloned into a pCR2.1-TOPO vector using the TOPO TA Cloning kit (Invitrogen), and transformed into E. coli TOPO10F' competent cells. The transformed culture was plated onto Luria-Bertani (LB) medium containing ampicillin/IPTG/X-Gal, and white colonies were screened for the insert fragment. Recombinant cultures were maintained in LB broth with glycerol at $-80{ }^{\circ} \mathrm{C}$ before screening.

Isolation of insert fragment by Southern blot analysis. To verify the presence of cloned inserts, white colonies were cultured in LB broth, and recombinant plasmid DNA was isolated using the QIAprep Spin Miniprep kit (Qiagen). Two-hundred and five inserts were amplified using primers directed at tester-ligated adaptor sequences in a GeneAmp PCR System 2700 (Applied Biosystems). Electrophoresis of each PCR product in $1.0 \%$ SeaKem LE agarose gel (FMC Bioproducts) was performed, and following ethidium bromide staining, the results were viewed under the Gel Doc 2000 imageanalysis system (Bio-Rad). PCR products were purified using a QIAquick PCR Purification kit (Qiagen), resuspended in $50 \mu \mathrm{l}$ distilled water, and denatured at $100{ }^{\circ} \mathrm{C}$ for $5 \mathrm{~min}$. Then, denatured DNA from each clone was dispensed into the separate wells of a BioDot apparatus (Bio-Rad) and transferred under vacuum onto Hybond-N + nylon membranes (Amersham Biosciences) which had been pre-equilibrated in $6 \times$ standard sodium citrate (SSC) (USB). The individual wells and membranes were washed with $2 \times$ SSC, and the membranes exposed to $1000 \mathrm{~mJ} \mathrm{UV}$ for $3 \mathrm{~min} 30 \mathrm{~s}$ in a Stratalinker (Stratagene). All hybridizations were performed at $42{ }^{\circ} \mathrm{C}$ after pre-hybridization in hybridization buffer at $42{ }^{\circ} \mathrm{C}$ for $2 \mathrm{~h}$. ECLlabelled DNA probes were prepared by the digestion of either $B$. anthracis CR or B. cereus KCTC $3624^{\mathrm{T}}$ genomic DNA with AluI/ HaeIII/RsaI in accordance with the ECL Direct nucleic acid labelling and detection system instructions (Amersham Biosciences). The membranes were washed twice at $20 \mathrm{~min}$ intervals in $0.5 \times \mathrm{SSC}+0.4 \%$ SDS, and $2 \times$ SSC at room temperature. Following high-stringency washing, the membranes were exposed to Kodak X-ray film MXG-1 (Kodak).

DNA sequencing analyses. Each clone was sequenced in duplicate with the M13 forward ( -20$)$ and/or reverse primers using the BigDye Terminator Cycle Sequencing kit and ABI PRISM 310 automated DNA sequencer (Applied Biosystems). Nucleotide sequence homologies of the genomic subtracted fragments of $B$. anthracis CR were determined using the BLAST suite of programs (Altschul et al., 1997) against the National Center for Biotechnology Information (NCBI) GenBank nonredundant database (http://www.ncbi.nlm.nih.gov).

PCR conditions for species identification. In order to develop rapid identification and diagnosis tools for $B$. anthracis, B. anthracis species-specific oligonucleotide primers based on the chromosomal glycosyltransferase group 1 family protein gene were designed using Primer 3 software (Rozen \& Skaletsky, 2000) and default settings. The glycosyltransferase group 1 family protein gene-based PCR was performed with $100 \mathrm{ng}$ genomic DNA template in $25 \mu$ l reaction mixtures containing $1 \mu \mathrm{M}$ each 20 bp primer, $2.5 \mu \mathrm{l}$ reaction buffer, $0.2 \mathrm{mM}$ dNTPs, $1.5 \mathrm{mM} \mathrm{MgCl}_{2}$ and $2.5 \mathrm{U}$ Taq polymerase (Roche Diagnostics). Amplification was carried out in a GeneAmp PCR system 2700 (Applied Biosystems) and primary PCR cycling conditions were: one cycle of initial denaturation at $94{ }^{\circ} \mathrm{C}$ for 5 min, followed by 35 cycles at $94{ }^{\circ} \mathrm{C}$ for $30 \mathrm{~s}, 64^{\circ} \mathrm{C}$ for $30 \mathrm{~s}$, and $72{ }^{\circ} \mathrm{C}$ for $30 \mathrm{~s}$, and one final extension at $72{ }^{\circ} \mathrm{C}$ for $7 \mathrm{~min}$. The amplicons were electrophoresed in $1.2 \%$ SeaKem LE agarose gel (FMC
Bioproducts) and visualized following ethidium bromide staining. The PCR assays were performed on eight B. anthracis, 33 B. cereus, 10 B. thuringiensis, six B. mycoides, one B. pseudomycoides, one $B$. weihenstephanensis and 19 closely related Bacillus strains.

\section{RESULTS AND DISCUSSION}

\section{Construction and screening of B. anthracis SSH libraries}

The search for genetic markers that enable the unambiguous differentiation of $B$. anthracis from other B. cereus group members is an important and challenging task. While there is extensive genetic diversity within $B$. cereus and B. thuringiensis, B. anthracis appears to be genetically clonal (Keim et al., 1997; Helgason et al., 2000). Unique genomic differences may assist in the understanding of genetic relationships between $B$. anthracis and its phylogenetic neighbours, and such differences may also function as DNA signatures that select for $B$. anthracis (Radnedge et al., 2003). This study has shown that $B$. anthracis can be discriminated from other $B$. cereus group taxa using a strategy based on SSH to develop primers specific to $B$. anthracis.

Two-hundred and five $B$. anthracis clones were obtained and screened for B. anthracis-specific sequences. Since the most important criteria for determining effective DNA signatures are their absence from closely related organisms (false-positive results) and their occurrence in all isolates of the target (false-negative results; Radnedge et al., 2003), clone libraries generated by SSH were tested by reverse Southern blot analysis using $B$. anthracis CR as the tester probe and B. cereus KCTC $3264^{\mathrm{T}}$ as the driver probe. Of these, 46 clones contained inserts that were undetectable using the driver probe but detectable using the tester probe, and dot-blot results of seven of the 46 positive clones are presented in Fig. 1. A comparison of the seven encircled wells shows that low levels of hybridization between the clones and the driver probe (Fig. 1a), indicated by faint signals, correspond with dense signals between the clones and the tester probe (Fig. 1b), where hybridization was high. These seven signals demonstrate the specificity of the $B$. anthracis $\mathrm{CR}$ probe and reflect the likelihood that these clones specifically contain chromosomal $B$. anthracis DNA. Similarly dense signals appearing in corresponding tester and driver wells are indicative of hybridization with both probes. They represent clones that harbour not only $B$. anthracis chromosomal DNA but also DNA inserts from $B$. cereus.

Only two of the 46 clones examined, $4.3 \%$, possessed sequences that were completely specific to chromosomal $B$. anthracis, a percentage that is slightly higher than the $1.1 \%$ determined by Dwyer et al. (2004) using SSH. It is possible that a pre-amplification step for both the driver and tester probes, prior to the initiation of the SSH procedure, results in the loss of some sequences. Also, if long DNA fragments are abundant in a restriction enzyme digest, they may form 
(a)

(b)
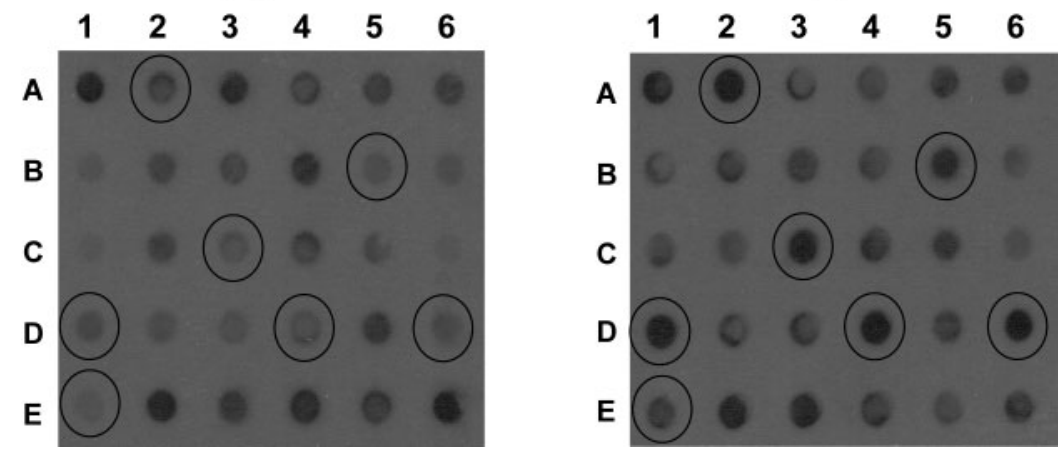

Fig. 1. Reverse Southern blot of PCR-amplified clones identified from $\mathrm{SSH}$ as specific to $B$. anthracis and hybridized to (a) the $B$. cereus KCTC $3624^{\top}$ probe and (b) the $B$. anthracis $\mathrm{CR}$ probe. Strong and weak signals represent high and low levels of hybridization, respectively, between clone and probe. Encircled wells represent seven clones that may contain B. anthracis-specific chromosomal DNA.

complex networks that prevent the formation of appropriate hybrids. In this study, the PCR-Select Bacterial Genome Subtractive Hybridization kit (Clontech) was employed, which uses a single enzyme, RsaI, for genomic digestion. It is possible that a more tailored $\mathrm{SSH}$ protocol that combines data from parallel SSH experiments using different restriction enzymes would have the potential to significantly increase the coverage of the genome compared with the use of a single enzyme. Nonetheless, it is likely that the low number of clones is simply a reflection of the homology that exists between $B$. anthracis and $B$. cereus group members. The remaining 44 clones contained sequences that either corresponded to $B$. anthracis-specific plasmids (pXO1, pXO2) or showed high nucleotide similarities, in excess of $90 \%$, with $B$. cereus and/or $B$. thuringiensis.

\section{Identification and annotation of $B$. anthracis- specific clones}

The results of the nucleotide sequence alignment analysis using NCBI BLAST are detailed in Table 2. Three nonsynthetic nucleotide sequence alignments were found, with GenBank accession numbers AE017334 (B. anthracis str. 'Ames Ancestor'), AE017225 (B. anthracis str. Sterne) and AE016879 (B. anthracis str. Ames). It appears that the 265 bp nucleotide sequence of the first $B$. anthracis-specific clone (clone 2/205) corresponds to part of the full $1389 \mathrm{bp}$ sequence of a conserved domain protein identified as belonging to a putative lysogenic prophage lambda $\mathrm{Ba} 03$. As would be expected of successful cloning, this sequence is unique to $B$. anthracis and is absent from all other members of the B. cereus group (Read et al., 2003). Indeed, Read et al. (2003) reported that 141 proteins in $B$. anthracis had no match to the protein set of the $B$. cereus ATCC 10987 sequence. It was suggested that these proteins were encoded by genes of unknown function and were possibly tranposases or, as appears to be the case for the 265 bp sequence of clone 2, were present in phage regions.

Comparative genome hybridization of $B$. anthracis and 19 members of the $B$. cereus group showed that they had $66-92 \%$ of chromosomal genes in common (Read et al.,
2003). Six small regions were identified as unique to the $B$. anthracis Ames strain genome, since they were absent from all other $B$. cereus group strains. Regions I-IV corresponded to $B$. anthracis prophages and, more specifically, region III contained the area designated as phage lambda $\mathrm{Ba} 03$. It is thought that bacteriophage sequences were acquired during or after the separation of B. anthracis from B. cereus and B. thuringiensis (Dwyer et al., 2004).

For clone 2, query coverage for all four significant sequence alignments versus the query sequence was $87 \%$, and the percentage identity between the query sequence and the aligned sub-sequences was 99\%, with expected values indicating that these four alignments were not a random occurrence (Table 2). This non-random occurrence also held true for all four significant sequence alignments observed with the second $B$. anthracis-specific clone (clone 5/205). Compared with clone 2 , clone 5 query coverage was much greater at 100\%, and maximum identities, $100 \%$, were expressed.

Clone 5 possessed a 617 bp sequence that corresponded to a portion of the full $1242 \mathrm{bp}$ sequence of the B. anthracis glycosyltransferase group I family protein gene. Table 2 shows that the three non-synthetic nucleotide sequence alignments identified in clone 5, GenBank accession numbers AE017334 (B. anthracis str. 'Ames Ancestor'), AE017225 (B. anthracis str. Sterne) and AE016879 (B. anthracis str. Ames) were present in clone 2 also. However, the synthetic nucleotide sequence alignments differed between clones 2 and 5. For clone 5, the synthetic construct B. anthracis clone FLH240894.01L (GenBank accession no. EF037225) was associated with the BA5519 gene for glycosyltransferase, and for clone 2, the synthetic construct B. anthracis clone FLH247209.01L (GenBank accession no. EF040076) was related to the BA4081 gene for a hypothetical protein.

An additional gene sequence appeared only upon full alignment of the 1242 bp nucleotide sequence, and it corresponded to B. cereus E33L (GenBank accession no. CP000001; data not shown). In this instance, the identity was much lower, $84 \%$, than for other sequence alignments. This level of dissimilarity was reinforced by the 203 base 


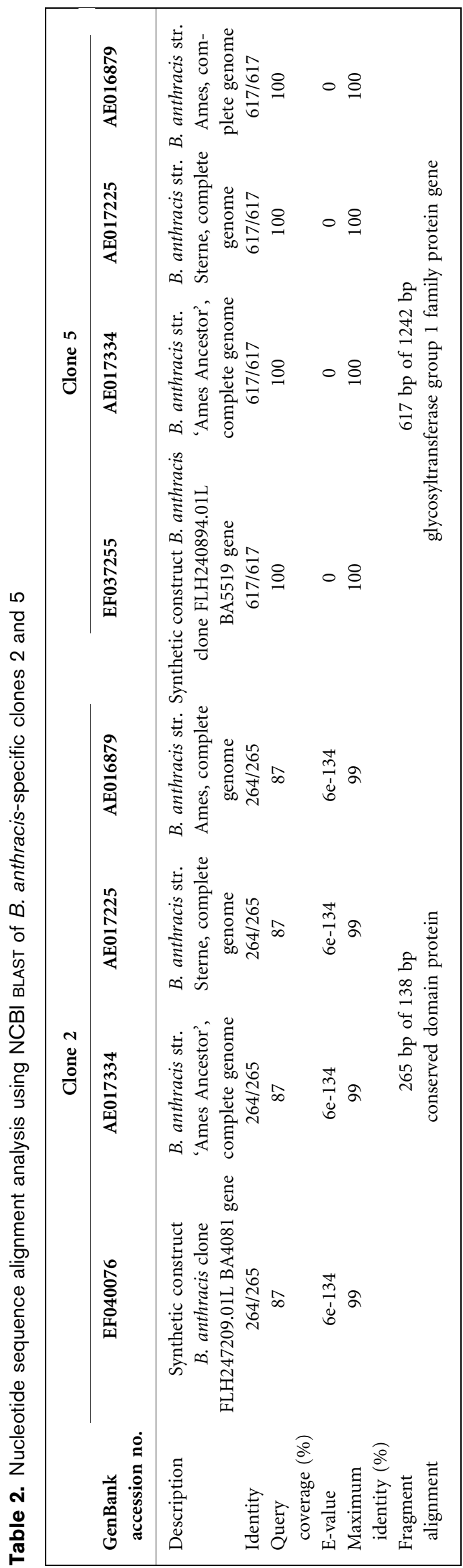

substitutions observed when both sequences were compared, and is less supportive of the reported high homology between $B$. anthracis and other B. cereus strains (Read et al., 2003; Bell et al., 2002; Helgason et al., 2000; Keim et al., 1997; Andersen et al., 1996). Interestingly, Han et al. (2006) sequenced the genomes of two isolates, $B$. thuringiensis 97 27 subsp. konkukian serotype H34, from a necrotic human wound, and B. cereus E33L, from a swab of a Zebra carcass in Namibia. It was shown by comparative amplified fragment length polymorphism (AFLP) analysis that of the two isolates, B. cereus E33L was more closely related to $B$. anthracis than to B. cereus and B. thuringiensis. This is in keeping with the concept that the $B$. cereus group has evolved as asexually derived clonal populations (Helgason et al., 2000; Priest et al., 2004), although B. thuringiensis 9727 subsp. konkukian serotype $\mathrm{H} 34$ and B. cereus E33L are members of the anthracis lineage (Han et al., 2006; Priest et al., 2004). This lineage also supports the molecular-based distinction between $B$. thuringiensis strains of commercial value and pathogenic strains of $B$. anthracis.

The 413 aa of the glycosyltransferase group I family protein of $B$. anthracis CR were aligned using NCBI BLASTP, and with expect values of 0 , shared $100 \%$ identity with amino acid sequences from B. anthracis str. Ames (GenBank accession no. AAP29164), B. anthracis str. 'Ames Ancestor' (GenBank accession no. AAT34661) and B. anthracis str. Sterne (GenBank accession no. AAT57416). Much lower amino acid sequence identities, for the expected value $0-2 \mathrm{e}^{-35}$, were observed between $B$. anthracis $C R$ and other bacteria, namely, B. cereus E33L (GenBank accession no. AAU15307; 80\%), Exiguobacterium sibiricum (GenBank accession no. EAM88130; 42\%), Thermoanaerobacter tengcongensis (GenBank accession no. AAM23928; 32\%), Clostridium botulinum (GenBank accession no. ABS34160; 29\%), Bacteroides fragilis (GenBank accession no. AAG26480; 29\%), and Geobacter metallireducens (GenBank accession no. EDJ80581; 27\%). Data reliability is enhanced because these measures ensure that more accurate E-values are generated that in turn distinguish more accurately between false and true matches (Gertz et al., 2006).

In an examination of genomic differences that distinguish B. anthracis from non-anthrax-causing Bacillus species, Radnedge et al. (2003) used a combination of AFLP and SSH and mapped $B$. anthracis sequences to five regions with distinct loci that were within $50 \mathrm{~kb}$ of each other on the $5.23 \mathrm{Mb}$ B. anthracis A2012 genome. However, none of the sequences detailed was purported to correspond to a glycosyltransferase group I family protein gene. More recently, Dwyer et al. (2004), identifying B. anthracis specific chromosomal sequences through the use of two SSH libraries, have found that half of the clones generated are located across regions I and II on the B. anthracis chromosome. Region I contains ORFs that share significant similarity with genes that encode the enzymes and proteins associated with cell wall polysaccharide biosynthesis. Typically, this process involves transferases that catalyse 
the sequential transfer of sugar residues forming the carbohydrate repeating structure of the cell wall.

Glycosyltransferases are involved in carbohydrate modification of the cell envelope, and this in turn may allow $B$. anthracis to modify its cell-surface characteristics. However, comparatively little is known of the carbohydrates in the vegetative cell walls of $B$. anthracis (Choudhury et al., 2006). Using aqueous hydrogen fluoride to isolate and structurally characterize Ames, Sterne and Pasteur strains of B. anthracis versus B. cereus ATCC 14579 and ATCC 10987, Choudhury et al. (2006) have shown that the cell wall structure of $B$. anthracis is clearly different from that of both $B$. cereus strains, and may have potential use as a target marker for the development of antimicrobials against anthrax.

Reviewing a comparative genome analysis of $B$. cereus group genomes with that of B. subtilis, Anderson et al. (2005) highlighted one species of UDP-glucose dehydrogenase in $B$. anthracis that is located in the same chromosomal cluster (BA5519-BA5512) as two genes that are homologous to a teichoic acid ATP binding cassette (ABC) transporter gene and, more significantly, a glycosyltransferase gene. This chromosomal cluster may encode the neutral cell wall polysaccharide characterized by Choudhury et al. (2006) that consists of an amino sugar backbone substituted with galactose residues, reflecting the more complex nature of this glycan mostly found in $B$. anthracis. In the current study, the clone 5 nucleotide sequence was aligned with GenBank accession number EF037225, a B. anthracis synthetic construct of the BA5519 gene. Therefore, the function of the glycosyltransferase sequence uncovered in this study may be associated with a capsular polysaccharide biosynthesis protein.

\section{Diagnostic screening of Bacillus strains using $B$. anthracis-specific, clone 2-specific primers}

Since the objective of this study was to use SSH to develop discriminating primers based on natural environmental strains of $B$. anthracis, attention focused on the sequence alignments with B. anthracis str. 'Ames Ancestor' (GenBank accession no. AE017334), B. anthracis str. Sterne (GenBank accession no. AE017225) and B. anthracis str. Ames (GenBank accession no. AE016879). Oligonucleotide primer construction for the species-specific discrimination of $B$. anthracis from other $B$. cereus group strains was based on information deduced from clone 5 and the full nucleotide sequence of the glycosyltransferase group I family protein gene. Two 20 bp primers, Ba-G206F (5'-TCTTCAGTGACAAAACCACA-3') and Ba-G1013R (5'-CAAGAAATCTTTTTCGAAGG-3'), were generated, and subsequently their specificities were tested against all 78 strains of the Bacillus species listed in Table 1.

Of the B. anthracis strains included in this analysis, six strains, ATCC $14578^{\mathrm{T}}$ and Korean isolates CR, BC, CAU 1, CAU 2 and CAU 3, harboured the pXO1 and pXO2 plasmids, and are therefore pathogenic to humans and animals. B. anthracis strains ATCC 14185 and ATCC 14186 harboured the pXO1 plasmid alone, rendering these strains avirulent but useful for vaccines. As seen in Fig. 2, the expected PCR product of 808 bp was common to all $B$. anthracis strains (lanes 1-8), regardless of virulence. This product was efficiently and distinctly detected, because no amplification of PCR products from any other closely related Bacillus strain occurred with primers Ba-G206F and Ba-G1013R.

The value of the SSH-derived primers used in this study is that they are discriminatory and are directed to the $B$. anthracis chromosome. This confers an advantage over primers that select for fragments of DNA located on plasmids pXO1 and pXO2, which can be lost from $B$. anthracis both in nature and in the laboratory. Bell et al. (2002) favoured the use of LightCycler PCR to select for the $B$. anthracis gene targets pagA and $p a g B$ on both virulence plasmids, and discouraged targeting chromosomal nucleic acid owing to the chromosomal homology among members of the B. cereus group. This contrasts with the decision of Keim et al. (1997), while assessing the molecular diversity of $B$. anthracis using AFLP, to ignore the presence or absence of plasmids pXO1 and pXO2 as discriminating genetic markers because of their ephemeral nature. Thus, reliance upon extrachromosomal markers alone may result in a failure to distinguish avirulent $B$. anthracis strains lacking both plasmids from near-neighbour $B$. cereus group strains, and therefore might lead to misidentification. Restriction digestion with AluI of an amplified SG-749 fragment revealed a restriction profile, of two DNA fragments, that was unique to $B$. anthracis, differentiating it from other strains of the $B$. cereus group (Daffonchio et al., 1999). The SG-850 fragment from which this marker was derived is common to $B$. cereus, $B$. thuringiensis and B. mycoides, as well as B. anthracis.

While arguments persist concerning reliance upon extrachromosomal markers to differentiate $B$. anthracis from other $B$. cereus group member strains, an interesting report by Hoffmaster et al. (2004) draws attention to the unusual B. cereus isolate G9241, confirmed by $16 \mathrm{~S}$ rRNA analysis, which can cause a severe inhalation anthrax-like illness. This isolate carries anthrax toxin genes on a circular plasmid, pBCXO1, and they share $99.6 \%$ homology with pXO2. In the current study, $20 \mathrm{~B}$. cereus Korean strains (WY 1-20) were isolated from patients with food poisoning, and all were negative when tested by PCR for the presence of $B$. anthracis plasmids. Although there were no atypical $B$. cereus strains among these Korean isolates, if $B$. cereus G9241 were shown to cause an anthrax-like gastrointestinal infection, it would be interesting to evaluate this and similar atypical B. cereus strains using the SSH-derived primers, Ba-G206F and Ba-G1013R.

$\mathrm{SSH}$ is the ideal, versatile method for two reasons. First, SSH allows for the swift cloning of cDNAs of differentially expressed genes because of their high level of enrichment, 


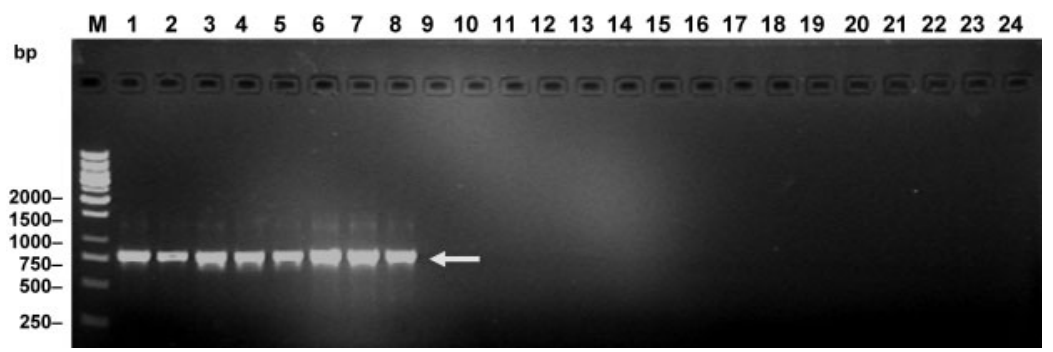

Fig. 2. Electrophoretic examination of $B$. anthracis-specific PCR products generated with primers Ba-G206F and Ba-G1013R based on the glycosyltransferase family 1 protein gene. Lanes: 1, GeneRuler 1 kb DNA Ladder (Fermentas); 2, B. anthracis ATCC $14578^{\top} ; 3$, B. anthracis ATCC 14185; 4, B. anthracis ATCC 14186; 5, B. anthracis CR; 6, B. anthracis BC; 7, B. anthracis CAU $1 ; 8$, B. anthracis CAU 2; 9, B. anthracis CAU 3; 10, B. cereus KCTC $3624^{\top} ; 11$, B. cereus KCTC 1012; 12, B. cereus KCTC $1013 ; 13$, B. cereus KCTC 1014; 14, B. cereus KCTC 1094; 15, B. cereus KCTC 1661; 16, B. cereus KCTC 3062; 17 , B. cereus ATCC 10702; 18, B. cereus ATCC 13061; 19, B. cereus ATCC 21769; 20, B. cereus ATCC 21771; 21, B. cereus ATCC $25621 ; 22$, B. cereus ATCC 27348; 23, B. cereus WY1; 24, B. cereus WY2. Data for the remaining 54 strains ( $n=78)$, including isolates from the $B$. cereus group and other Bacillus species, are not shown because the results for these strains are the same as those seen in lanes 9-24; no $B$. anthracis-specific DNA is present. The arrow shows the expected PCR product of 808 bp common to all $B$. anthracis strains (lanes 1-8).

low background and normalized abundance of cDNAs in the subtracted mixture. Second, it is possible to use uncloned subtracted cDNA mixtures as hybridization probes (Diatchenko et al., 1996). In addition, the application of SSH is wide-ranging. Although it has been used to identify genetic differences between virulent and avirulent pathogens such as Mycobacterium ulcerans (Jenkin et al., 2003), Mycoplasma agalactiae and Mycoplasma bovis (Marenda et al., 2004, 2005), and Helicobacter pylori (Oleastro et al., 2006), it has also been used as a tool to identify genetic diversity in environmental microbial communities (Galbraith et al., 2004).

This study used SSH to develop primers Ba-G206F and BaG1013R for the successful discrimination of B. anthracis from other $B$. cereus group members. These new primers offer the opportunity for rapid, optimal screening in the routine diagnosis of presumptive $B$. anthracis. The time and cost advantages over other DNA-based methods have been outlined previously, but work is still needed to elucidate the nature and role of the glycosyltransferase group I family protein gene identified in this study.

\section{ACKNOWLEDGEMENTS}

This work was supported by the 21C Frontier Microbial Genomics and Application Center Program, Ministry of Science and Technology (grant MG05-0102-2-0) and the Korea Health 21 R\&D Project, Ministry of Health and Welfare (grant A060767), Republic of Korea. We are grateful to Dr Elayne Ferguson (UK) for providing editorial assistance.

\section{REFERENCES}

Agron, P. G., Macht, M., Radnedge, L., Skowronski, E. W., Miller, W. \& Andersen, G. L. (2002). Use of subtractive hybridization for comprehensive surveys of prokaryotic genome differences. FEMS Microbiol Lett 211, 175-182.

Altschul, S. F., Madden, T. L., Schaffer, A. A., Zhang, J., Zhang, Z., Miller, W. \& Lipman, D. J. (1997). Gapped BLAST and PSI-BLAST: a new generation of protein database search programs. Nucleic Acids Res 25, 3389-3402.

Andersen, G. L., Simchock, J. M. \& Wilson, K. H. (1996). Identification of a region of genetic variability among Bacillus anthracis strains and related species. J Bacteriol 178, 377-384.

Anderson, l., Sorokin, A., Kapatral, K., Reznik, G., Bhattacharya, A., Mikhailova, N., Burd, H., Joukov, V., Kaznadzey, D. \& other authors (2005). Comparative genome analysis of Bacillus cereus group genomes and Bacillus subtilis. FEMS Microbiol Lett 250, 175-184.

Ash, C. \& Collins, M. D. (1992). Comparative analysis of 23 S ribosomal RNA gene sequences of Bacillus anthracis and emetic Bacillus cereus determined by PCR-direct sequencing. FEMS Microbiol Lett 73, 75-80.

Ash, C., Farrow, J. A., Dorsch, M., Stackebrandt, E. \& Collins, M. D. (1991). Comparative analysis of Bacillus anthracis, Bacillus cereus, and related species on the basis of reverse transcriptase sequencing of $16 \mathrm{~S}$ rRNA. Int J Syst Bacteriol 41, 343-346.

Ausubel, F. M., Brent, R., Kingston, R. E., Moore, D. D., Seidman, J. G. \& Struhl, K. (1993). Current Protocols in Molecular Biology, Section 2.4. New York: John Wiley and Sons.

Bell, C. A., Uhl, J. A., Hadfield, T. L., David, J. C., Meyer, R. F., Smith, T. F. \& Cockerill, F. R., III (2002). Detection of Bacillus anthracis DNA by LightCycler PCR. J Clin Microbiol 40, 2897-2902.

Carlson, C. R., Caugant, D. A. \& Kolsto, A. B. (1994). Genotypic diversity among Bacillus cereus and Bacillus thuringiensis strains. Appl Environ Microbiol 60, 1719-1725.

Cherif, A., Borin, S., Rizzi, A., Ouzari, H., Boudabous, A. \& Daffonchio, D. (2003). Bacillus anthracis diverges from related clades of the Bacillus cereus group in 16S-23S ribosomal DNA intergenic transcribed spacers containing tRNA genes. Appl Environ Microbiol 69, 33-40.

Choudhury, B., Leoff, C., Saile, E., Wilkins, P., Quinn, C. P., Kannenberg, E. L. \& Carlson, R. W. (2006). The structure of the major cell wall polysaccharide of Bacillus anthracis is species-specific. J Biol Chem 281, 27932-27941. 
Daffonchio, D., Borin, S., Consolandi, A., Mora, D., Manachini, P. L. \& Sorlini, C. (1998). 16S-23S rRNA internal transcribed spacers as molecular markers for the species of the $16 \mathrm{~S}$ rRNA group I of the genus Bacillus. FEMS Microbiol Lett 163, 229-236.

Daffonchio, D., Borin, S., Frova, G., Gallo, R., Mori, E., Fani, R. \& Sorlini, C. (1999). A randomly amplified polymorphic DNA marker specific for the Bacillus cereus group is diagnostic for Bacillus anthracis. Appl Environ Microbiol 65, 1298-1303.

Diatchenko, L., Lau, Y. F., Campbell, A. P., Chenchik, A., Moqadam, F., Huang, B., Lukyanov, S., Lukyanov, K., Gurskaya, N. \& other authors (1996). Suppression subtractive hybridization: a method for generating differentially regulated or tissue-specific cDNA probes and libraries. Proc Natl Acad Sci U S A 93, 6025-6030.

Dwyer, K. G., Lamonica, J. M., Schumacher, J. A., Williams, L. E., Bishara, J., Lewandowski, A., Redkar, R., Patra, G. \& DelVecchio, V. G. (2004). Identification of Bacillus anthracis specific chromosomal sequences by suppressive subtractive hybridization. BMC Genomics 5, 15 .

Ellerbrok, H., Nattermann, H., Ozel, M., Beutin, L., Appel, B. \& Pauli, G. (2002). Rapid and sensitive identification of pathogenic and apathogenic Bacillus anthracis by real-time PCR. FEMS Microbiol Lett 214, 51-59.

Galbraith, E. A., Antonopoulos, D. A. \& White, B. A. (2004). Suppressive subtractive hybridization as a tool for identifying genetic diversity in an environmental metagenome: the rumen as a model. Environ Microbiol 6, 928-937.

Gertz, E. M., Yu, Y.-K., Agarwala, R., SchAffer, A. C. \& Altschul, S. F. (2006). Composition-based statistics and translated nucleotide searches: improving the TBLASTN module of BLAST. BMC Biol 4, 41.

Han, C. S., Xie, G., Challacombe, J. F., Altherr, M. R., Bhotika, S. S., Brown, N., Bruce, B., Campbell, C. S., Campbell, M. L. \& other authors (2006). Pathogenomic sequence analysis of Bacillus cereus and Bacillus thuringiensis isolates closely related to Bacillus anthracis. J Bacteriol 188, 3382-3390.

Harrell, L. J., Andersen, G. L. \& Wilson, K. H. (1995). Genetic variability of Bacillus anthracis and related species. J Clin Microbiol 33, 1847-1850.

Helgason, E., Økstad, O. A., Caugant, D. A., Johansen, H. A., Fouet, A., Mock, M., Hegna, I. \& KolstA,, A. B. (2000). Bacillus anthracis, Bacillus cereus, and Bacillus thuringiensis - one species on the basis of genetic evidence. Appl Environ Microbiol 66, 2627-2630.

Helgason, E., Tourasse, N. J., Meisal, R., Caugant, D. A. \& Kolstø, A. B. (2004). Multilocus sequence typing for bacteria of the Bacillus cereus group. Appl Environ Microbiol 70, 191-201.

Hoffmaster, A. R., Ravel, J., Rasko, D. R., Chapman, G. D., Chute, M. D., Marston, C. K., De, K. B., Sacchi, C. T., Fitzgerald, C. \& other authors (2004). Identification of anthrax toxin genes in a Bacillus cereus associated with an illness resembling inhalation anthrax. Proc Natl Acad Sci U S A 101, 8449-8454.

Jenkin, G. A., Stinear, T. P., Johnson, D. R. \& Davies, J. K. (2003). Subtractive hybridization reveals a type I polyketide synthase locus specific to Mycobacterium ulcerans. J Bacteriol 185, 6870-6882.

Jones, H., Ostrowski, M. \& Scanlan, D. J. (2006). A suppression subtractive hybridization approach reveals niche-specific genes that may be involved in predator avoidance in marine Synechococcus isolates. Appl Environ Microbiol 72, 2730-2737.

Keim, P. \& Smith, K. L. (2002). Bacillus anthracis evolution and epidemiology. Curr Top Microbiol Immunol 271, 21-32.

Keim, P., Kalif, A., Schupp, J., Hill, K., Travis, S. E., Richmond, K., Adair, D. M., Hugh-Jones, M., Kuske, C. R. \& other authors (1997). Molecular evolution and diversity in Bacillus anthracis as detected by amplified fragment length polymorphism markers. J Bacteriol 179, 818-824.

Kim, W., Hong, Y. P., Yoo, J. H., Lee, W. B., Choi, C. S. \& Chung, S. I. (2002). Genetic relationships of Bacillus anthracis and closely related species based on variable-number tandem repeat analysis and BOXPCR genomic fingerprinting. FEMS Microbiol Lett 207, 21-27.

Lechner, S., Mayr, R., Francis, K. P., Pruss, B. M., Kaplan, T., Gunkel, E. W., Stewart, G. S. A. B. \& Scherer, S. (1998). Bacillus weihenstephanensis sp. nov. is a new psychrotolerant species of the Bacillus cereus group. Int J Syst Bacteriol 48, 1373-1382.

Logan, N. A., Carman, J. A., Melling, J. \& Berkeley, R. C. (1985). Identification of Bacillus anthracis by API tests. J Med Microbiol 20, 75-85.

Marenda, M. S., Vilei, E. M., Poumarat, F., Frey, J. \& Berthelot, X. (2004). Validation of the suppressive subtractive hybridization method in Mycobacterium agalactiae species by the comparison of a field strain with the type strain PG2. Vet Res 35, 199-212.

Marenda, M. S., Sagné, E., Poumarat, F. \& Citti, C. (2005). Suppression subtractive hybridization as a basis to assess Mycoplasma agalactiae and Mycoplasma bovis genome diversity and species-specific sequences. Microbiology 151, 475-489.

Mohammed, M. J., Marston, C. K., Popovic, T., Weyant, R. S. \& Tenover, F. C. (2002). Antimicrobial susceptibility testing of Bacillus anthracis: comparison of results obtained using the National Committee for Clinical Laboratory Standards broth microdilution references and Etest agar gradient diffusion methods. J Clin Microbiol 40, 1902-1907.

Nakamura, L. K. (1998). Bacillus pseudomycoides sp. nov. Int J Syst Bacteriol 48, 1031-1035.

Odendaal, M. W., Pieterson, P. M., de Vros, V. \& Botha, A. D. (1991). The biochemical, morphological and virulence profiles of Bacillus anthracis isolated in the Kruger National Park. Onderstepoort J Vet Res 58, 21-26.

Oleastro, M., Monteiro, L., Lehours, P., Mégraud, F. \& Ménard, A. (2006). Identification of markers for Helicobacter pylori strains isolated from children with peptic ulcer disease by suppressive subtractive hybridization. Infect Immun 74, 4064-4074.

Priest, F. G., Barker, M., Baillie, L. W., Holmes, E. C. \& Maiden, M. C. (2004). Population structure and evolution of the Bacillus cereus group. J Bacteriol 186, 7959-7970.

Radnedge, L., Agron, P. G., Hill, K. K., Jackson, P. J., Ticknor, L. O., Keim, P. \& Andersen, G. L. (2003). Genome differences that distinguish Bacillus anthracis from Bacillus cereus and Bacillus thuringiensis. Appl Environ Microbiol 69, 2755-2764.

Read, T. D., Peterson, S. N., Tourasse, N., Baillie, L. W., Paulsen, I. T., Nelson, K. E., Tettelin, H., Fouts, D. E., Eisen, J. A. \& other authors (2003). The genome sequence of Bacillus anthracis Ames and comparison to closely related bacteria. Nature 423, 81-86.

Rozen, S. \& Skaletsky, H. J. (2000). Primer3 on the WWW for general users and for biologist programmers. In Bioinformatics Methods and Protocols: Methods in Molecular Biology, pp. 365-386. Edited by S. Krawetz \& S. Misener. Totowa, NJ: Humana Press.

Sastry, K. S., Tuteja, U., Santhosh, P. K., Lalitha, M. K. \& Batra, H. V. (2003). Identification of Bacillus anthracis by a simple protective antigen-specific mAb dot-ELISA. J Med Microbiol 52, 47-49.

Schuch, R., Nelson, D. \& Fischetti, V. A. (2002). A bacteriolytic agent that detects and kills Bacillus anthracis. Nature 418, 884-889.

Volokhov, D., Pomerantsev, A., Kivovich, V., Rasooly, A. \& Chizhikov, V. (2004). Identification of Bacillus anthracis by multiprobe microarray hybridization. Diagn Microbiol Infect Dis 49, 163-171. 